\title{
Front Matter: Volume 9273
}

, "Front Matter: Volume 9273," Proc. SPIE 9273, Optoelectronic Imaging and Multimedia Technology III, 927301 (19 December 2014); doi:

10.1117/12.2179419

SPIE. Event: SPIE/COS Photonics Asia, 2014, Beijing, China 


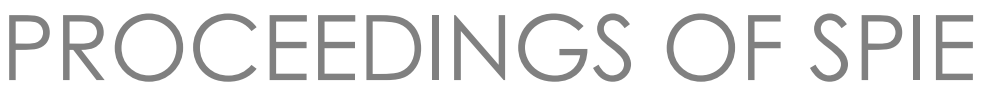

\title{
Optoelectronic Imaging and Multimedia Technology III
}

\author{
Qionghai Dai \\ Tsutomu Shimura \\ Editors
}

\section{9-11 October 2014 Beijing, China}

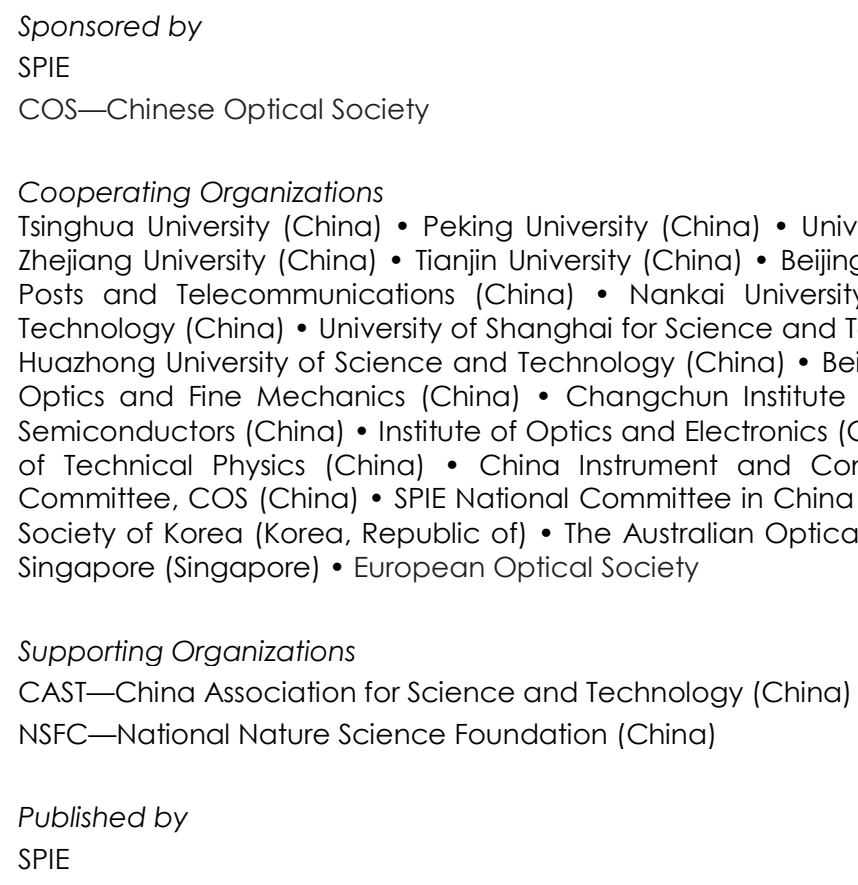
Singapore (Singapore) • European Optical Society

Supporting Organizations

CAST_China Association for Science and Technology (China)

NSFC-National Nature Science Foundation (China)

Published by

SPIE Technology (China) • University of Shanghai for Science and Technology (China) • Capital Normal University (China) Huazhong University of Science and Technology (China) • Beijing Jiaotong University (China) • Shanghai Institute of Optics and Fine Mechanics (China) - Changchun Institute of Optics and Fine Mechanics (China) - Institute of Semiconductors (China) • Institute of Optics and Electronics (China) • Institute of Physics (China) • Shanghai Institute of Technical Physics (China) - China Instrument and Control Society (China) - Optoelectronics Technology Committee, COS (China) • SPIE National Committee in China (China) • Optical Society of Japan (Japan) • Optical Society of Korea (Korea, Republic of) - The Australian Optical Society (Australia) - Optics and Photonics Society of

Volume 9273

Proceedings of SPIE 0277-786-786X, V.9273

SPIE is an international society advancing an interdisciplinary approach to the science and application of light.

Optoelectronic Imaging and Multimedia Technology III, edited by Qionghai Dai, Tsutomu Shimura, Proc. of SPIE Vol. 9273, $927301 \cdot$ ( ) 2014 SPIE · CCC code: 0277-786X/14/\$18

doi: $10.1117 / 12.2179419$

Proc. of SPIE Vol. 9273 927301-1 
The papers included in this volume were part of the technical conference cited on the cover and title page. Papers were selected and subject to review by the editors and conference program committee. Some conference presentations may not be available for publication. The papers published in these proceedings reflect the work and thoughts of the authors and are published herein as submitted. The publisher is not responsible for the validity of the information or for any outcomes resulting from reliance thereon.

Please use the following format to cite material from this book:

Author(s), "Title of Paper," in Optoelectronic Imaging and Multimedia Technology III, edited by Qionghai Dai, Tsutomu Shimura, Proceedings of SPIE Vol. 9273 (SPIE, Bellingham, WA, 2014) Article CID Number.

ISSN: 0277-786X

ISBN: 9781628413465

Published by

SPIE

P.O. Box 10, Bellingham, Washington 98227-0010 USA

Telephone +1 3606763290 (Pacific Time) · Fax +1 3606471445

SPIE.org

Copyright (C) 2014, Society of Photo-Optical Instrumentation Engineers.

Copying of material in this book for internal or personal use, or for the internal or personal use of specific clients, beyond the fair use provisions granted by the U.S. Copyright Law is authorized by SPIE subject to payment of copying fees. The Transactional Reporting Service base fee for this volume is $\$ 18.00$ per article (or portion thereof), which should be paid directly to the Copyright Clearance Center (CCC), 222 Rosewood Drive, Danvers, MA 01923. Payment may also be made electronically through CCC Online at copyright.com. Other copying for republication, resale, advertising or promotion, or any form of systematic or multiple reproduction of any material in this book is prohibited except with permission in writing from the publisher. The CCC fee code is 0277-786X/14/\$18.00.

Printed in the United States of America.

Publication of record for individual papers is online in the SPIE Digital Library.

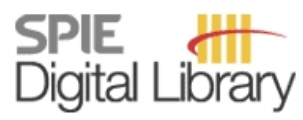

SPIEDigitalLibrary.org

Paper Numbering: Proceedings of SPIE follow an e-First publication model, with papers published first online and then in print and on CD-ROM. Papers are published as they are submitted and meet publication criteria. A unique, consistent, permanent citation identifier (CID) number is assigned to each article at the time of the first publication. Utilization of CIDs allows articles to be fully citable as soon as they are published online, and connects the same identifier to all online, print, and electronic versions of the publication. SPIE uses a six-digit CID article numbering system in which:

- The first four digits correspond to the SPIE volume number.

- The last two digits indicate publication order within the volume using a Base 36 numbering system employing both numerals and letters. These two-number sets start with 00, 01, 02, 03, $04,05,06,07,08,09,0 A, 0 B \ldots 0 Z$, followed by 10-1Z, 20-2Z, etc.

The CID number appears on each page of the manuscript. The complete citation is used on the first page, and an abbreviated version on subsequent pages. Numbers in the index correspond to the last two digits of the six-digit CID number. 


\title{
Contents
}

\author{
ix Authors \\ xiii Symposium Committees \\ xv Conference Committee
}

\section{OPTICAL INFORMATION PROCESSING}

927307 Illuminant spectrum estimation using a digital color camera and a color chart [9273-1]

927309 High dynamic range coding imaging system [9273-3]

9273 OB Compressive photography based on lens array with coded mask [9273-5]

9273 OC A model of PSF estimation for coded mask infrared imaging [9273-6]

\section{MULTISPECTRAL AND HYPERSPECTRAL IMAGING}

$9273 \mathrm{OE} \quad$ Real-time automatic small infrared target detection using local spectral filtering in the frequency [9273-8]

9273 OF Texture-adaptive hyperspectral video acquisition system with a spatial light modulator [9273-9]

9273 OG Color correction of underwater images using spectral data [9273-10]

$9273 \mathrm{OH} \quad$ Non-negative structural sparse representation for high resolution hyperspectral imaging [9273-11]

HIGH-SPEED AND HIGH-RESOLUTION IMAGING

9273 0J Implementing two compressed sensing algorithms on GPU [9273-15]

9273 OK High-resolution light field cameras based on a hybrid imaging system [9273-17]

\section{D IMAGE/VIDEO SYSTEMS}

9273 OM A semi-automatic 2D-to-3D video conversion with adaptive key-frame selection [9273-19]

9273 ON Joint bit allocation for 3D video coding based on virtual view distortion [9273-47] 
927300 A depth video processing algorithm for high encoding and rendering performance [9273-21]

$9273 \mathrm{OP} \quad$ A novel virtual viewpoint merging method based on machine learning [9273-22]

$92730 Q \quad$ A foreground object features-based stereoscopic image visual comfort assessment model [9273-23]

\section{MACHINE VISION METHODS, ARCHITECTURES, AND APPLICATIONS}

9273 OR Unsupervised abnormal crowd activity detection using interaction power model [9273-24]

9273 OS A crude to fine method to detect salient region [9273-25]

9273 OT Tablet-based two-dimensional measurement for estimating the embryo area of brown rice [9273-26]

9273 OU A vein display system based on three-dimensional reconstruction [9273-27]

9273 OV Detection for processing history of seam insertion and contrast enhancement [9273-28]

COMPUTATIONAL IMAGING

9273 OY Light-field-based phase imaging [9273-31]

$92730 Z$ Light field creating and imaging with different order intensity derivatives [9273-32]

IMAGE/VIDEO ANALYSIS, PROCESSING, AND RETRIEVAL

927311 A compression method of Els in integral imaging [9273-37]

927312 Full-reference quality assessment of stereoscopic images by learning sparse monocular and binocular features [9273-38]

927313 Optical image encryption based on a modified radial shearing interferometer [9273-46]

TIME-OF-FLIGHT IMAGING

927314 Computational imaging of light in flight (Invited Paper) [9273-40]

927315 Partial scene reconstruction using time-of-flight imaging [9273-41]

927316 Three-dimensional patterning in transparent materials with spatiotemporally focused femtosecond laser pulses [9273-42]

927318 Depth map super-resolution and enhancement for time-of-flight cameras [9273-44] 
$92731 \mathrm{~A}$ Image super-resolution via adaptive filtering and regularization [9273-16]

9273 1B Light field reconstruction robust to signal dependent noise [9273-33]

9273 1C An improved opto-digital joint transform correlator reducing the influence of defocus on image motion measurement [9273-45]

$92731 \mathrm{E} \quad$ Accurate point spread function (PSF) estimation for coded aperture cameras [9273-52]

9273 IF Non-intrusive gesture recognition system combining with face detection based on Hidden Markov Model [9273-53]

$92731 \mathrm{G}$ Oil tank detection based on salient region and geometric features [9273-54]

$92731 \mathrm{H} \quad$ Experimental calibration of x-ray camera performance: spatial resolution, flat field response, and radiation sensitivity [9273-55]

$927311 \quad$ An example image super-resolution algorithm based on modified k-means with hybrid particle swarm optimization [9273-56]

9273 1K Multimodal visual dictionary learning via heterogeneous latent semantic sparse coding [9273-58]

$92731 \mathrm{~L} \quad$ Magnifying arbitrarily selected areas of fractal Chinese characters [9273-59]

9273 1M Adaptive block size selection for inter-layer backward view synthesis prediction [9273-60]

9273 IN Phase recovery based on quadratic programming [9273-62]

$927310 \quad$ Laser 3D imaging technology based on digital micromirror device and the performance analysis [9273-63]

$92731 Q \quad$ Kernel-based discriminant image filter learning: application in face recognition [9273-65]

9273 IR An effective representation for action recognition with human skeleton joints [9273-66]

9273 is A combined ASEF and pictorial structure method for facial landmark detection [9273-67]

$92731 \mathrm{U} \quad$ Image segmentation using an improved differential algorithm [9273-69]

$92731 \mathrm{M} \quad$ Modeling of polarimetric BRDF characteristics of painted surfaces [9273-72]

$92731 \mathrm{Y} \quad$ A three-dimensional shape measurement system based on fiber optic image bundles [9273-73]

$92731 \mathrm{Z}$ Image fusion driven by the analysis of sparse coefficients [9273-74] 
927320 Synthesis multi-projector content for multi-projector three dimension display using a layered representation [9273-75]

927322 Coupled data association and $/ 1$ minimization for multiple object tracking under occlusion [9273-78]

927323 Hierarchical feature selection for erythema severity estimation [9273-79]

927324 Automatic segmentation of psoriasis lesions [9273-80]

927325 3D reconstruction of large target by range gated laser imaging [9273-81]

927326 A no-reference contourlet-decomposition-based image quality assessment method for super-resolution reconstruction [9273-82]

927327 Real-time remote three-dimensional superresolution range-gated imaging based on interframe correlation [9273-84]

927328 Improved sequential search algorithms for classification in hyperspectral remote sensing images [9273-85]

$927329 \quad$ Image feature point detection method based on the pixels of high-resolution sensors [9273-86]

9273 2C Asymmetric multiview image coding based on feature matching [9273-90]

$92732 \mathrm{D} \quad$ High quality underwater imaging platform with laser range gated technique combining with image denoising and restoration [9273-92]

92732 G High dynamic range algorithm based on HSI color space [9273-95]

$92732 \mathrm{H} \quad$ Amplitude and phase of single nanoparticle calculated using finite-difference timedomain method [9273-96]

$927321 \quad$ Combining heterogeneous features for 3D hand-held object recognition [9273-97]

9273 2J An auto-gain control algorithm for EMCCD based on dynamic gray-level [9273-98]

$92732 \mathrm{~K} \quad$ A flexible design for coded aperture snapshot spectral imager [9273-99]

$92732 \mathrm{~L} \quad$ High accuracy hole filling for Kinect depth maps [9273-100]

9273 2M Orientation selectivity based structure for texture classification [9273-101]

927320 Realization of a single image haze removal system based on DaVinci DM6467T processor [9273-103]

$92732 \mathrm{P} \quad$ Embedding perspective cue in holographic projection display by virtual variable-focallength lenses [9273-104]

$92732 \mathrm{Q}$ Simultaneous cartoon-plus-texture image deconvolution by using variational image decomposition [9273-105] 
$92732 \mathrm{R} \quad$ Applications of just-noticeable depth difference model in joint multiview video plus depth coding [9273-106]

$92732 \mathrm{~T}$ Characteristic extraction and matching algorithms of ballistic missile in near-space by hyperspectral image analysis [9273-108]

$92732 \mathrm{U} \quad$ Ultrasonic televiewer image encoding based on block prediction [9273-109]

92732 V Seismic data compression based on wavelet transform [9273-110]

9273 2W A design method of the distortionless catadioptric panoramic imaging based on freeform surface [9273-111]

$92732 X \quad$ Compression of multispectral image using HEVC [9273-112]

$92732 Y$ Design of HD binocular stereo display system based on ARM11 [9273-113]

$927322 \quad$ A new metric to assess temporal coherence for video retargeting [9273-114]

927330 An effective guess for Gerchberg-Saxton-type algorithms [9273-115]

927331 Improved grey world color correction method based on weighted gain coefficients [9273-116]

927333 Compressive spectral video acquisition with double-channel complementary coded aperture [9273-118]

927334 Aircraft detection based on probability model of structural elements [9273-119]

927335 Efficient stereo matching algorithm with edge-detecting [9273-120]

927336 Multi-channel super-resolution with Fourier ptychographic microscopy [9273-121]

927337 Efficient mode decision algorithm for scalable high efficiency video coding [9273-122]

927338 Explore spatial-temporal relations: transient super-resolution with PMD sensors [9273-123]

927339 Auto-calibration for multi-projector 3D display [9273-124]

9273 3A A large-scale glasses-free 3D display system using multiple projectors [9273-125]

9273 3B Motion blurred image restoration based on joint transform correlator [9273-126]

9273 3C Image reconstruction in Fizeau interferometry [9273-127]

9273 3D Image reconstruction in speckle interferometry [9273-128]

9273 3E Remote stereoscopic video play platform for naked eyes based on the Android system [9273-129]

$92733 \mathrm{~F}$ Study of phase retrieval algorithm from partially coherent light [9273-130] 
9273 3G Application of time-resolved glucose concentration photoacoustic signals based on an improved wavelet denoising [9273-131]

$92733 \mathrm{H} \quad$ High-emulation mask recognition with high-resolution hyperspectral video capture system [9273-132]

$927331 \quad$ Image restoration based on wavelets and curvelet [9273-133]

9273 3J Accurate 3D reconstruction using a multi-phase ToF camera [9273-134]

9273 3K Excitation of topological insulator plasmons by two-dimensional periodic structure [9273-136]

9273 3L Improvement on the polynomial modeling of digital camera colorimetric characterization [9273-137] 
Li, Deng-hui, 3B

Li, Guangyu, $\mathrm{OH}$

Li, Guo, 33

Li, Hong, IY

Li, Houqiang, 1M, IR

Li, Huan, 2K, 33

Li, Jiafeng, OE

Li, Jianwei, OV

Li, Ke, $2 Z$

Li, Kemeng, 12

$\mathrm{Li}$, Linbo, $1 \mathrm{H}$

Li, Ming-gao, IF, 10

Li, Panpan, 37

$\mathrm{Li}$, Shoufeng, $3 \mathrm{H}$

Li, Sining, 25

Li, Xiangyang, 2

Li, Xiao Bing, 3K

Li, Xuesong, 11

Li, Yangguang, 18

Li, Youfu, 27

Li, Zeren, 2D

Li, Zhaohui, 2P

Li, Zhenhua, 2D, 2Q

Liang, Ding, 3J

Liang, Xiaoyue, $1 Y$

Lin, Jingyu, 38

Lin, Shengnan, OR

Lin, Weisi, $2 M$

Lin, Xing, 38

Liv, Chao, 2R

Liu, Danhua, 2K, 33

Liu, Guodong, $3 G$

Liu, Huan, $2 \mathrm{H}$

Liu, Huiping, OG

Liu, Jing, 35, 3E

Liu, Jingdan, OY

Liu, Jingjing, 2J

Liu, Meiqin, $2 \mathrm{C}$

Liu, Ming, 2G

Liu, Qiang, $1 \mathrm{~K}$

Liu, Shihua, 2T

Liu, Xiaohua, 2G

Liv, Xiaoquan, 27

Liu, Xingchun, 29

Liu, Yangdong, 35

Liu, Yebin, 3J

Liu, Zhuang, 20

Lu, Dajiang, 13

Lu, Dezhen, 27

Lu, Jing, OK

LU, Li, 2T

Lu, Liu, 2M

LU, Wei Bing, 3K

Lu, Wei, 25

Lu, Yuanzhi, 11

Luo, Xiaoyan, 1Z, 31

LV, Peijun, IY

Lv, Xiong, 21

Ma, Jinlong, $1 \mathrm{E}$

Ma, Ran, 37
Ma, Yike, OK

Meng, Guiyu, $\mathrm{OH}$

Nakariyakul, Songyot, 28

$\mathrm{Ni}$, Jielei, 16

$\mathrm{Ni}, \mathrm{Na}, 1 \mathrm{~N}$

$\mathrm{Ni}$, Rongrong, OV

Ning, Yang, 24

Pan, Bin, 31

Peng, Xiang, 13

Peng, Zongju, 0O, OP, OQ

Pongsoon, Prasit, OT

Prasertsak, Anchalee, OT

Qian, Yi-xian, 3B

Qian, Yuehong, 2J

Qin, Chen, 20

Qu, Lei, 1Q, 1S

Ren, Bin, 20

Ren, Jingbo, 1 A

Ren, Kun, 1B

Ren, Zhong, $3 G$

Sang, Xinzhu, 35, 3E

Shao, Feng, $0 Q, 12$

Shen, Jun-fei, 2W

Sheng, Chengkai, 00

Sheng, Wen, 2T

Shi, Chenbo, 23, 24

Shi, Guangming, $\mathrm{OH}, 1 \mathrm{~A}, 2 \mathrm{~K}, 2 \mathrm{M}, 33$

Shi, Guozhong, 35

Shi, Junsheng, 07, 2Y, 3L

Shi, Nandi, 37

Shi, Xiao-wei, 3B

Shi, Yujiao, $1 \mathrm{U}$

Shu, Chang, 23, 24

Situ, Guohai, OY

Sugioka, Koji, 16

Sumriddetchkajorn, Sarun, OT

Sun, Mingui, OE, OR, OS

Sun, Yi, OC, $1 \mathrm{E}$

Suo, Jinli, 1B

Tai, Yonghang, 07, 2Y, 3L

Tan, Jiubin, 11

Tang, Shao, 39, 3A

Tao, Wenjun, 2C

Wang, Chao-Yan, 3C

Wang, Danting, OU

Wang, Haogian, OB

Wang, Helong, OS

Wang, Hui, OP

Wang, Jian, 3K

Wang, Jianxin, $0 N, 2 L$

Wang, Jincheng, $0 G$

Wang, Li, 23, 24

Wang, Lizhi, 2K

Wang, Qing, OC

Wang, Qing, 22

Wang, Rongbo, 2D

Wang, Shigang, 11

Wang, Shouyu, 2D

Wang, Shuang, 2l

Wang, Wei, 25 
Wang, Xiaorui, 2P

Wang, Xiaoyan, 11

Wang, Xingzheng, OB

Wang, Xinwei, 27

Wang, Xue, 22

Wang, Yan, 15

Wang, Yongjin, OF, 3H

Wang, Yu, OZ

Wang, Yuan-qing, 1F, 10

Wang, Zeying, $1 \mathrm{X}$

Wang, Zhaohui, 16

Wang, Zhe, 29

Wei, Jingxuan, $1 \mathrm{C}$

Wei, Kaiyun, 30

Wei, Ping, OJ

Wei, Sui, 1Q, 1S, 3F

Wei, Zhang, 3F

WU, Dongmei, $1 U$

Wu, Hao, $1 \mathrm{~A}$

Wu, Jinjian, $2 M$

Wu, Junfeng, 31

Wu, Renfan, 09

Wu, Yi-si, 2W

Wu, Zhaoguo, oU

Xie, Hongwei, $1 \mathrm{H}$

Xie, Xiaopeng, $1 \mathrm{C}$

Xiong, Hongkai, OM, 15

$\mathrm{Xu}$, Liujing, $1 \mathrm{~F}$

$X \cup$, Ning, $1 \mathrm{~L}$

$X \cup$, Tingfa, OY

Xue, Ya-lan, 10

Yan, Bo, $2 Z$

Yan, Chenggang, 2X, 38, 3J

Yan, Keding, 2D, 2Q

Yan, Liv, 3F

Yan, Wei, 3J

Yan, $\mathrm{Xu}, 25$

Yan, Zhengang, 2D

Yang, Chao, ON

Yang, Jingyu, OC, $1 \mathrm{E}$

Yang, Yang, 3l

Yang, YU, OG

Yao, Yuan, IG

Yi, Hongwei, $1 \mathrm{C}$

Ying, $\mathrm{H}_{\text {., }} \mathrm{OQ}$

You, Zhixiang, $2 \mathrm{~L}$

Yu, Hongfei, 07, 3L

Yu, Jia, OG

Yu, Mei, OQ, 12

Yu, Xiujuan, 12

Yuan, Binhang, $2 Z$

Yuan, Ding, OE, OR, $1 Z$

Yue, Weirui, OY

Yun, Lijun, $2 Y$

Zeng, Bin, 16

Zhang, Ao, OC

Zhang, Quan Bing, $1 \mathrm{~N}$

Zhang, Dayong, 25

Zhang, Faqiang, $1 \mathrm{H}$

Zhang, Haopeng, 1G, 31
Zhang, Hong, OE, OR, OS

Zhang, Jiancheng, 29, $2 \mathrm{G}$

Zhang, Jianqi, $2 P$

Zhang, Jun, $2 Q$

Zhang, Lei, 18, 39, 3A

Zhang, Lingchen, 1Q

Zhang, Qian, 37

Zhang, Wei, 1L, 2U, 2V

Zhang, Wei, 26

Zhang, Wenwen, 2J

Zhang, Xianzhi, 2T

Zhang, Yazhong, 2M

Zhang, Ying, $1 \mathrm{X}$

Zhang, Yongbing, 18, 36, 39, 3A

Zhang, Yongdong, OK

Zhang, Yuchen, 15

Zhang, Zhaoyang, ON, 2L, 2R

Zhang, Zhengbing, 1L, 2U, 2V

Zhao, Fuliang, $2 \mathrm{P}$

Zhao, Hanwen, $1 Z$

Zhao, Hui, 1C

Zhao, Huijie, $1 X, 1 Y$

Zhao, Yao, OV, 2C

Zhao, Yuejin, 2G

Zhen, Cheng, IY

Zheng, Di, OP

Zheng, Zhen-rong, 2W

Zhou, Bi-ye, IF, 10

Zhou, Jile, $1 \mathrm{~K}$

Zhou, Tong, 11

Zhou, Wengang, IR

Zhou, Ya, OU

Zhou, Yan, 27

Zhuo, Bin, $2 Y$

Zuo, Yifan, 2L, 2R 
Proc. of SPIE Vol. $9273927301-12$

Downloaded From: https://www.spiedigitallibrary.org/conference-proceedings-of-spie on 26 Apr 2023 Terms of Use: https://www.spiedigitallibrary.org/terms-of-use 


\title{
Symposium Committees
}

\author{
General Chairs
}

H. Philip Stahl, NASA Marshall Space Flight Center (USA)

Bingkun Zhou, Tsinghua University (China)

General Co-chairs

Arthur Chiou, National Yang-Ming University (Taiwan, China)

Jianlin Cao, China Ministry of Science and Technology (China)

Junhao Chu, Shanghai Institute of Technical Physics (China)

Technical Program Chairs

Songlin Zhuang, Shanghai University of Science and Technology (China)

Xingde Li, Johns Hopkins University (United States)

Technical Program Co-chairs

Qiming Wang, Institute of Semiconductors (China)

Xu Liu, Zhejiang University (China)

Daoyin Yu, Tianjin University (China)

Qihuang Gong, Peking University (China)

Tianchu Li, National Institute of Metrology (China)

Wei Huang, Nanjing University of Posts and Telecommunications (China)

Local Organizing Committee Chair

Guangcan Guo, University of Science and Technology of China (China) 
Local Organizing Committee Co-chairs

Guoqiang Ni, Beijing Institute of Technology (China)

Shusen Xie, Fujian Normal University (China)

Xiaomin Ren, Beijing University of Posts and Telecommunications (China)

Ying Gu, People's Liberation Army General Hospital (China)

Huilin Jiang, Changchun University of Science and Technology (China)

General Secretary

Qihuang Gong, Peking University (China)

Local Organizing Committee

Yan Li, Chinese Optical Society/Peking University (China)

Zhiping Zhou, Peking University (China)

Changhe Zhou, Shanghai Institute of Optics and Fine Mechanics (China)

Qingming Luo, Huazhong University of Science and Technology (China)

Chongxiu Yu, Beijing University of Posts and Telecommunications (China)

Hongda Chen, Institute of Semiconductors (China)

Yongtian Wang, Beijing Institute of Technology (China)

Yiping Cui, Southeast University (China)

Xuping Zhang, Nanjing University (China)

Feijun Song, Daheng Corporation (China)

Cunlin Zhang, Capital Normal University (China)

Yanting Lu, Nanjing University (China)

Yuejin Zhao, Beijing Institute of Technology (China)

Chunqing Gao, Beijing Institute of Technology (China)

Tiegen Liu, Tianjin University (China)

Xiaocong Yuan, Nankai University (China)

Weimin Chen, Chongqing University (China)

Zhongwei Fan, Academy of Optoelectronics (China)

Hanyi Zhang, Tsinghua University (China)

Lan Wu, Zhejiang University (China)

Yongsheng Zhang, University of Science and Technology of China (China)

Hong Yang, Peking University (China)

Xiaoying Li, Tianjin University (China)

Wei Xiong, Chinese Optical Society (China) 


\title{
Conference Committee
}

\author{
Conference Chairs
}

Qionghai Dai, Tsinghua University (China)

Tsutomu Shimura, The University of Tokyo (Japan)

\section{Conference Program Committee}

Moshe Ben-Ezra, MIT Media Laboratory (United States)

Xudong Chen, National University of Singapore (Singapore)

Ya Cheng, Shanghai Institute of Optics and Fine Mechanics (China)

Jinwei Gu, Huawei Technologies Co., Ltd. (United States)

Yo-Sung Ho, Gwangju Institute of Science and Technology

(Korea, Republic of)

Bormin Huang, University of Wisconsin-Madison (United States)

Matthias B. Hullin, Universität Bonn (Germany)

Ivo Ihrke, INRIA Bordeaux (France)

Yoshiaki Kanamori, Tohoku University (Japan)

Chun-Chieh Jay Kuo, The University of Southern California

(United States)

Kyros Kutulakos, University of Toronto (Canada)

Wanqing Li, University of Wollongong (Australia)

Yuan Luo, National Taiwan University (Taiwan, China)

Imari Sato, National Institute of Informatics (Japan) and Tokyo

Institute of Technology (Japan)

Yoichi Sato, The University of Tokyo (Japan)

John T. Sheridan, University College Dublin (Ireland)

Guangming Shi, Xidian University (China)

Lei Tian, University of California, Berkeley (United States)

Feng Wu, University of Science and Technology of China (China)

Bo Yang, University of Shanghai for Science and Technology (China)

Xiaolin Zhang, Shanghai Institute of Microsystem and Information

Technology (China)

Zhenrong Zheng, Zhejiang University (China)

\section{Session Chairs}

Keynote Session I

Qionghai Dai, Tsinghua University (China)

Keynote Session II

Qionghai Dai, Tsinghua University (China) 
Keynote Session III

George Barbastathis, Massachusetts Institute of Technology (United States)

Optical Information Processing

Chun-Chieh Jay Kuo, The University of Southern California (United States)

Multispectral and Hyperspectral Imaging

Laura Waller, University of California, Berkeley (United States)

High-Speed and High-Resolution Imaging

Wenjie Wan, Shanghai Jiao Tong University (China)

3D Image/Video Systems

Yuan Luo, National Taiwan University (Taiwan, China)

Machine Vision Methods, Architectures, and Applications

Yebin Liu, Tsinghua University (China)

Computational Imaging

Jingyi Yu, University of Delaware (United States)

Image/Video Analysis, Processing, and Retrieval

Wolfgang Heidrich, The University of British Columbia (Canada)

Time-of-Flight Imaging

Matthias B. Hullin, Universität Bonn (Germany) 